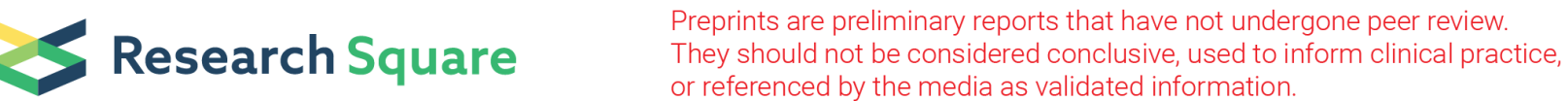

\section{Correlation Between Disability Measure and Black Hole (T1 Hypointense Lesion) Lesion Load in Brain Magnetic Resonance Imaging (MRI) of Patients With Multiple Sclerosis (MS): Protocol for a Systematic Review and Meta-analysis}

\section{Amir Valizadeh}

Tehran University of Medical Sciences https://orcid.org/0000-0001-5983-8527

Elham Barati

Tehran University of Medical Sciences

Mohammad Ali Sahraian

Tehran University of Medical Sciences https://orcid.org/0000-0002-3224-8807

Mohammad Reza Fattahi

Tehran University of Medical Sciences

Mana Moassefi ( $\nabla$ Moassefi@gmail.com )

Tehran University of Medical Sciences, Building no.1, Northern gate of the university

https://orcid.org/0000-0002-0111-7791

\section{Method Article}

Keywords: Multiple Sclerosis, Magnetic Resonance Imaging

Posted Date: February 22nd, 2021

DOI: https://doi.org/10.21203/rs.3.rs-194103/v3

License: (c) (i) This work is licensed under a Creative Commons Attribution 4.0 International License.

Read Full License 


\section{Abstract}

Introduction: As the role of neurodegeneration in the pathophysiology of multiple sclerosis (MS) has become more prominent, the formation and evolution of chronic or persistent T1-hypointense lesions (Black Holes) have been used as markers of axonal loss and neuronal destruction to measure disease activity. However, findings regarding this subject are controversial. In this study we aim to clarify the level of importance of T1 hypointense lesions for estimating the prognosis of patients.

Methods: We will search MEDLINE (through PubMed), Embase and Web of Science for relevant studies. We will extract the Spearman's rank correlation coefficient (SRCC) between the T1 hypointense lesion volume and Extended Disability Status Scale (EDSS) in participants. All included studies will be evaluated for the risk of bias. We will also perform a meta-analysis on the data. The risk of publication bias will be evaluated using Funnel plots. Finally, we will assess the confidence in cumulative evidence using an adapted version of GRADE.

\section{Introduction}

\section{Rationale}

Multiple sclerosis (MS) is a chronic, disabling disease that targets patients in work productive years. Prognostic factors play important roles in evaluating the cost of the disease on both individual patients and the social economy. Since there is no exact method to predict MS progression confidently, four general MS phenotypes have been defined: clinically isolated syndrome (CIS), relapsing-remitting MS (PRMS), secondary progressive (SPMS), and primary progressive (PPMS) [2,3]. RRMS is the most common MS phenotype. It is characterized by noticeable exacerbation or initiation of neurologic symptoms. These attacks, also called relapses are followed by periods of recovery, called remissions. Some patients who are diagnosed with RRMS will gradually convert to a secondary progressive course which is a progressive deterioration of neurologic function over time. The other two types are not mentioned in this study.

Magnetic resonance imaging (MRI) is a sensitive para clinical test for diagnosis and assessment of disease progression in MS and is often used to evaluate therapeutic efficacy. As the role of neurodegeneration in the pathophysiology of MS has become more prominent, the formation and evolution of chronic or persistent T1-hypointense lesions (black holes) have been used as markers of axonal loss and neuronal destruction to measure disease activity. On the one hand, T1 lesion load showed greater cross-sectional [4] and longitudinal [4, 5] correlations with Expanded Disability Status Scale (EDSS[6]) scores (a scale extensively used in studies for the assessment of disability for patients with MS) for patients with RRMS or SPMS than did T2 lesion load. On the other hand, several other studies reported an absence of correlations between T1 hypointensity and EDSS in patients with SPMS [7-11] and RRMS $[9,12]$. 
In this study, we aim to review all the relevant studies, to evaluate the correlation between T1 hypointensities (Black holes) on brain MRI with disability level of the patients with MS. Our main goal is to clarify if this possible prognostic factor can be used to help to determine the prognosis of patients.

\section{Objectives}

To evaluate the correlation between T1 hypointensities (Black holes) lesion load (lesion mean volume) on brain MRI with disability level of patients with RRMS or SPMS.

\section{Methods}

Design and methods used for this protocol comply with Centre for Reviews and Dissemination (CRD's) Guidance For Undertaking Reviews in Healthcare [13] and is reported in line with Preferred Reporting Items for Systematic Reviews and Meta-Analyses Protocols (PRISMA-P) [14]. Eligibility criteria were informed using the PICOTS [15] system.

\section{Eligibility criteria}

(P) Population: adult patients diagnosed with Relapsing-Remitting Multiple Sclerosis (PRMS) or Secondary Progressive Multiple Sclerosis (SPMS), based on the McDonald criteria [2, 16-18]:

- Two attacks or symptom flare-ups (lasting at least 24 hours with 30 days between attacks), plus two lesions.

- Two attacks, one lesion, and evidence of dissemination in space (or a different attack in a different part of the nervous system).

- One attack, two lesions, and evidence of dissemination in time (or finding a new lesion - in the same location - since the previous scan, or presence of immunoglobulin, called oligoclonal bands in the spinal fluid).

- One attack, one lesion, and evidence of dissemination in space and time.

- Worsening of symptoms or lesions and dissemination in space found in two of the following: MRI of the brain, MRI of the spine, and spinal fluid.

- Relapsing-Remitting_course: A multiple sclerosis course characterized by relapses with stable neurological disability between episodes.

- Progressive course: A multiple sclerosis course characterized by steadily increasing objectively documented neurological disability independent of relapses. Fluctuations, periods of stability, and superimposed relapses might occur. Primary progressive multiple sclerosis (a progressive course from disease onset) and secondary progressive multiple sclerosis (a progressive course following an initial relapsing-remitting course) are distinguished. 
(I) Index (Prognostic factor): T1 hypointense (black hole) lesion mean volume (lesion load) on brain Magnetic Resonance Imaging (MRI)

(C) Comparator: not applicable

(0) Outcome: disability measure using Expanded Disability Status Scale (EDSS)

(T) Timing: measured at the same time MRI was performed (or with a very close time interval between)

(S) Setting: any

\section{Information sources}

The search will employ sensitive topic-based strategies designed for each database with no time frame limitations. There will be no language or geographical restrictions either. We will perform our search on the $10^{\text {th }}$ of February, 2021.

Databases:

- MEDLINE through PubMed

- Embase

- Science Citation Index - Expanded (Web of Science)

- Conference Proceedings Citation Index - Science (Web of Science)

\section{Search strategy}

Our search strategies for all the databases included in our study, namely MEDLINE (through PubMed), Embase, Science Citation Index - Expanded (Web of Science), and Conference Proceedings Citation Index - Science (Web of Science) are presented in appendix A.

\section{Study records}

\section{Data management}

Records will be managed through EndNote version X9 [19]; specific software for managing bibliographies. 


\section{Selection process}

Two reviewers (AV and MM) will independently screen the title and abstract of identified studies for inclusion. We will link publications from the same study to avoid including data from the same study more than once. If any study cannot be clearly excluded based on its title and abstract, its full text will be reviewed. A study will be included when both reviewers independently assess it as satisfying the inclusion criteria from the full text. A third reviewer (MF) will act as arbitrator in the event of disagreement following discussion. We will prepare a flow diagram of the number of studies identified and excluded at each stage in accordance with the PRISMA flow diagram of study selection [1].

\section{Data collection process}

Using a standardized form, two reviewers (AV and MM) will extract the data independently. We will resolve any disagreements by discussion or, if required, by consultation with a third review author (MF). We will attempt to extract data presented only in graphs and figures whenever possible but will include such data only if two reviewers independently obtain the same result. If studies are multi-center, then where possible we will extract data relevant to each. In the case of missingness of data, if possible, we will try to contact the original investigators to request missing information. In case that was unsuccessful, we will only analyze the available information and will not try to impute any missing data.

\section{Data items}

Data extracted will include the following summary data: sample characteristics, sample size, study methods, inclusion and exclusion criteria, MRI settings used, founding sources, declarations of interests, and results.

\section{Outcomes and prioritization}

Our main outcome of interest is the relationship between participants' EDSS score and T1 hypointense lesion mean volume.

\section{Risk of bias in individual studies}

Two review authors (AV and MM) will assess the risk of bias of each included study. We will resolve any disagreements by consensus, or by consultation with a third review author (MF). Because at the moment there is no standard tool for assessing the risk of bias in overall prognosis studies, we will use a tailored version of the Quality In Prognosis Studies (QUIPS) tool for assessing the risk of bias in studies [20], presented in Appendix B. Our tailored version of the tool consists of six risks of bias domains: study participation, study attrition, prognostic factor measurement, outcome measurement, study confounding, 
and statistical analysis and reporting. The study participation domain consists of five items: an adequate description of the source population or population of interest, adequate description of the baseline study sample, adequate description of the sampling frame and recruitment, adequate description of the period and place of recruitment, and adequate description of inclusion and exclusion criteria. The study attrition domain consists of four items: description of attempts to collect information on participants who dropped out, reasons for loss to follow-up provided, an adequate description of participants lost to followup, and no important differences between participants who completed the study and those who did not. The prognostic factor measurement domain consists of two items: provision of clear definition or description of the prognostic factor, and reporting of continuous variables or use of appropriate cut points. The outcome measurement domain consists of three items: provision of a clear definition of the outcome, use of an adequately valid and reliable method of outcome measurement, and use of same method and setting of outcome measurement in all study participants. The study confounding domain consists of the seven items: measurement of all important confounders, provision of clear definitions of the important confounders measured, adequately valid and reliable measurement of all important confounders, use of same method, and setting of confounding measurement in all study participants, appropriate imputation methods used for missing confounders (if applicable), important potential confounders accounted for in the study design, and important potential confounders accounted for in the analysis. The statistical analysis and reporting domain consists of two items: sufficient presentation of data to assess the adequacy of the analytic strategy, and an adequate statistical model for the design of the study.

\section{Data synthesis}

We will use R version 4 [21] as the software for our data synthesis. We expect correlation coefficients ( $r$ ) to be our primary outcome measure. Most meta-analysts do not perform syntheses on the correlation coefficient itself because the variance depends strongly on the correlation. Rather, the correlation is converted to the Fisher's z scale and all analyses are performed using the transformed values [22]. For the meta-analysis of correlation data, we first convert the correlation coefficients to Fisher's $z$ scale. Then we will calculate the variance and standard error of the Fisher's z. We will perform a meta-analysis on those values based on the random-effects model. Finally, we will convert back Fisher's $z$ to correlation coefficient ( $r$ ) for the sake of presentation.

\section{Assessment of heterogeneity}

We expect some heterogeneity between studies because of ethnicity and methodological diversity. We will report the range of the effects of the random-effects meta-analyses using prediction intervals. In a random-effects meta-analysis, the prediction interval reflects the whole distribution of effects across study populations, including the effect expected in a future study [23, 24]. 


\section{Subgroup analysis}

If at least 5 studies are available for each classification of MS in our study (RRMS and SPMS), we will perform a subgroup analysis for each of them.

\section{Sensitivity analysis}

We plan to perform sensitivity analyses to explore the influence of the following factors:

- Studies at high or unclear risk of bias

- Very long or large studies to establish the extent to which they dominate the results.

\section{Meta-bias}

To evaluate the risk of reporting bias across studies, a test for funnel plot asymmetry will be conducted. This test examines whether the relationship between estimated effect size and study size is greater than chance [25]. Funnel plots will be generated for visual inspection of potential publication bias. In the presence of publication bias, the plot will be symmetrical at the top, and data points will increasingly be missing from the middle to the bottom parts of the plot [26].

\section{Confidence in cumulative evidence}

The strength of the overall body of evidence will be assessed using an adapted version of the Grading of Recommendations, Assessment, Development and Evaluation (GRADE) framework for prognostic factor research [27], which takes into account seven criteria: study limitations, inconsistency, indirectness, imprecision, publication bias, moderate/large effect size, and dose effect. Two review authors (AV and $\mathrm{MM}$ ) rate the certainty of the evidence for the outcome as 'high', 'moderate', 'low', or 'very low'. We resolve any discrepancies by consensus, or, if needed, by arbitration by a third review author (MF).

\section{Appendices}

\section{Appendix A}

\section{Embase}

\#8 \#7 AND \#1

\#7 \#6 AND \#5 
\#6 disability:ab,ti,kw

\#5 \#2 OR \#3 OR \#4

\#4 'black hole*':ab,ti,kw

\#3 (t1 NEAR/5 hypointens $\left.{ }^{\star}\right): a b, t i, k w$

\#2 (t1 NEAR/5 lesion*):ab,ti,kw

\#1 'multiple sclerosis'/exp

\section{Medline through PubMed}

Multiple Sclerosis[MeSH Terms] AND ((disability[tiab]) AND ("T1 hypointens*"[tiab] OR "T1 lesion*"[tiab] OR "black hole*"[tiab]))

\section{CENTRAL}

\#1 MeSH descriptor: [Multiple Sclerosis, Relapsing-Remitting] explode all trees

\#2 MeSH descriptor: [Multiple Sclerosis, Chronic Progressive] explode all trees

\#3 \#1 OR \#2

\#4 (t1 NEAR/5 lesion*):ab,ti,kw

\#5 (t1 NEAR/5 hypointens*):ab,ti,kw

\#6 'black hole*':ab,ti,kw

\#7 \#4 OR \#5 OR \#6

\#8 disability:ab,ti,kw

\#9 \#8 AND \#7

\#10 \#9 AND \#3

\section{Web of Science}

\#1 TS="Multiple Sclerosis" 
\#2 TS=(t1 NEAR/5 lesion*)

\#3 TS=(t1 NEAR/5 hypointens*)

\#4 TS="black hole*"

\#5 \#2 OR \#3 OR \#4

\#6 TS="disability"

\#7 \#6 AND \#5

\#8 \#7 AND \#1

\section{Appendix B}

Our tailored version of the Quality In Prognosis Studies (QUIPS) 


\section{Study participation: yes/no/partly/unsure/NA}

a. Adequate participation in the study by eligible individuals

b. Adequate description of the source population or population of interest

c. Adequate description of the baseline study sample

d. Adequate description of the sampling frame and recruitment

e. Adequate description of the period and place of recruitment

\section{f. Adequate description of} inclusion and exclusion criteria

Study participation: risk of bias rating (high/low/unclear)
NA: usually participants with information on T1 hypointensity in brain MRI and information on disability levels or relapse rate are selected from a greater study cohort

The source population for the cohort with T1 hypointensity in MRI is clearly described

Age and number of participants at baseline is clearly described

Way of establishing the source population, selection criteria, and key characteristics of the source population clearly described

The period and place of recruitment for both baseline and follow-up visits are clearly described

Criteria used for diagnosis and classification and description of other inclusion and exclusion criteria are clearly described

High: most items are answered with 'no'; Low: all items answered with 'yes'; Unclear: most items are answered with 'unsure' Note: potentially a single item may introduce a high risk of bias, depending on study specifics

\section{Study attrition: yes/no/partly/unsure/NA}

a. Adequate response rate for study participants

b. Attempts to collect information on participants who dropped out described

c. Reasons for loss to followup provided

\section{d. Adequate description of} participants lost to follow-up

e. No important differences between participants who completed the study and those who did not

Study attrition: risk of bias rating (high/low/unclear)
NA: usually participants with information on T1 hypointensity in brain MRI and information on disability levels or relapse rate are selected from a greater study cohort

Attempts to collect information on participants who dropped out are described (e.g., telephone contact, mail, registers)

Reasons on participants who dropped out are available (e.g., deceased participants between baseline and follow-up, participants moving to another location)

Key characteristics of participants lost to follow-up are described (T1 hypointensity load)

Study authors described differences between participants completing the study and those who did not as not important or information provided to judge the differences

High: most items are answered with 'no'; Low: all items answered with 'yes'; Unclear: most items are answered with 'unsure' Note: potentially a single item may introduce a high risk of bias, depending on study specifics 
a. Clear definition or description provided

b. Adequately valid and reliable method of measurement

c. Continuous variables reported or appropriate cutpoints used

d. Same method and setting of measurement used in all study participants

e. Adequate proportion of the study sample had complete data

f. Appropriate methods of imputation were used for missing data

Prognostic factor measurement: risk of bias rating (high/low/ unclear)
Study authors clearly indicate how they measure T1 hypointense lesions

NA: brain MRI is the only available method for measuring T1 hypointense lesions

The exact size of the T1 hypointense lesion is reported and it is not categorized base on cut points

NA: brain MRI is the only available method for measuring T1 hypointense lesions

NA: usually participants with information on T1 hypointensity in brain MRI and information on disability levels or relapse rate are selected from a greater study cohort

NA: missing radiological size of T1 hypointense lesion can't be imputed

High: most items are answered with 'no'; Low: all items answered with 'yes'; Unclear: most items are answered with 'unsure' Note: potentially a single item may introduce a high risk of bias, depending on study specifics

\section{Outcome measurement: yes/no/partly/unsure/NA}
a. Clear definition of the
Measuring disability level using EDSS should be clearly defined outcome provided

b. Use of an adequately valid and reliable method of

EDSS should be measured by a trained physician

outcome measurement

c. Use of same method and setting of outcome

Measurements of EDSS are the same for all participants measurement in all study participants

Outcome measurement: risk of bias rating (high/low/unclear)

High: most items are answered with 'no'; Low: all items answered with 'yes'; Unclear: most items are answered with 'unsure' Note: potentially a single item may introduce a high risk of bias, depending on study specifics

\section{Study confounding: yes/no/partly/unsure/NA}

\section{a. Measurement of all} important confounders

b. Provision of clear definitions of the important confounders measured

c. Adequately valid and
Important confounders are: ethnicity, comedications, and comorbidities, socioeconomic status

Measurement of confounders has to be clearly described 
d. Use of same method and setting of confounding measurement in all study participants

e. Appropriate imputation methods used for missing confounders (if applicable)

f. Important potential confounders were accounted for in the study design

g. Important potential confounders were accounted for in the analysis

\section{Study confounding} measurement: risk of bias rating (high/ low/unclear)
Measurements of confounders are the same for all study participants
Strategy to impute missing confounder data is described

Methods section of the publication describes the strategy to account for confounders

Important confounders are accounted for in multivariable logistic regression and Cox proportional hazards models

\section{Statistical analysis and reporting: yes/no/partly/unsure/NA}

a. Sufficient presentation of data to assess the adequacy of the analytic strategy

b. Strategy for model building is appropriate and based on a conceptual framework or model

c. Statistical model is adequate for the study design

d. No selective reporting of results

Statistical analysis and reporting: risk of bias rating (high/ low/unclear)
Mean or median values, including confidence intervals or standard errors or standard deviations

NA: we do not anticipate conceptual frameworks or explicit model building strategies for this type of research question (focusing on one prognostic factor only)

Mainly correlation coefficients
NA: EDSS score and relapse rate are the only outcomes; if missing the study will be excluded

No: no or no relevant information to answer the signaling question

Partly: information is provided but not completely

Unsure: not enough information to answer signaling question with yes or no

NA (not applicable): signaling question not appropriate for this type of prognostic review 
1. Moher, D., et al., Preferred reporting items for systematic reviews and meta-analyses: the PRISMA statement. PLoS medicine, 2009. 6(7): p. e1000097.

2. Thompson, A.J., et al., Diagnosis of multiple sclerosis: 2017 revisions of the McDonald criteria. Lancet Neurol, 2018. 17(2): p. 162-173.

3. Lublin, F.D., et al., Defining the clinical course of multiple sclerosis: the 2013 revisions. Neurology, 2014. 83(3): p. 278-86.

4. Truyen, L., et al., Accumulation of hypointense lesions ("black holes") on T1 spin-echo MRI correlates with disease progression in multiple sclerosis. Neurology, 1996. 47(6): p. 1469-1476.

5. Paolillo, A., et al., Quantitative MRI in patients with secondary progressive MS treated with monoclonal antibody Campath 1H. Neurology, 1999. 53(4): p. 751-7.

6. Cao, H., et al., Automatic Assessment of Expanded Disability Status Scale (EDSS) in Multiple Sclerosis Using a Decision Tree. Engineering, 2013. 05(10): p. 566-569.

7. Giugni, E., et al., MRI measures and their relations with clinical disability in relapsing-remitting and secondary progressive multiple sclerosis. Multiple Sclerosis Journal, 1997. 3(4): p. 221-225.

8. O'Riordan, J.I., et al., T1 hypointense lesion load in secondary progressive multiple sclerosis: a comparison of pre versus post contrast loads and of manual versus semi automated threshold techniques for lesion segmentation. Mult Scler, 1998. 4(5): p. 408-12.

9. Simon, J.H., et al., A longitudinal study of T1 hypointense lesions in relapsing MS: MSCRG trial of interferon beta-1a. Multiple Sclerosis Collaborative Research Group. Neurology, 2000. 55(2): p. 18592.

10. Koudriavtseva, T., et al., Gadolinium enhanced MRI predicts clinical and MRI disease activity in relapsing-remitting multiple sclerosis. J Neurol Neurosurg Psychiatry, 1997. 62(3): p. 285-7.

11. Masek, M., et al., Secondary-progressive form of multiple sclerosis: MRI changes versus clinical status. Neuro Endocrinol Lett, 2008. 29(4): p. 461-6.

12. van Walderveen, M.A., et al., Correlating MRI and clinical disease activity in multiple sclerosis: relevance of hypointense lesions on short-TR/short-TE (T1-weighted) spin-echo images. Neurology, 1995. 45(9): p. 1684-90.

13. Reviews, C.f. and Dissemination, CRD's guidance for undertaking reviews in healthcare. 2009: York Publ. Services.

14. Moher, D., et al., Preferred reporting items for systematic review and meta-analysis protocols (PRISMA-P) 2015 statement. Systematic reviews, 2015. 4(1): p. 1-9.

15. Moons, K.G., et al., Critical appraisal and data extraction for systematic reviews of prediction modelling studies: the CHARMS checklist. PLoS Med, 2014. 11(10): p. e1001744.

16. Polman, C.H., et al., Diagnostic criteria for multiple sclerosis: 2005 revisions to the "McDonald Criteria". Annals of Neurology, 2005. 58(6): p. 840-846.

17. McDonald, W.I., et al., Recommended diagnostic criteria for multiple sclerosis: guidelines from the International Panel on the diagnosis of multiple sclerosis. Ann Neurol, 2001. 50(1): p. 121-7. 
18. Polman, C.H., et al., Diagnostic criteria for multiple sclerosis: 2010 revisions to the McDonald criteria. Ann Neurol, 2011. 69(2): p. 292-302.

19. Hupe, M., EndNote X9. Journal of Electronic Resources in Medical Libraries, 2019. 16(3-4): p. 117119.

20. Hayden, J.A., P. Cote, and C. Bombardier, Evaluation of the quality of prognosis studies in systematic reviews. Ann Intern Med, 2006. 144(6): p. 427-37.

21. Team, R.C., R: A language and environment for statistical computing. 2013.

22. Rosenblad, A., Introduction to Meta-Analysis by Michael Borenstein, Larry V. Hedges, Julian PT Higgins, Hannah R. Rothstein. 2009, Wiley Online Library.

23. IntHout, J., et al., Plea for routinely presenting prediction intervals in meta-analysis. BMJ Open, 2016. 6(7): p. e010247.

24. Riley, R.D., J.P. Higgins, and J.J. Deeks, Interpretation of random effects meta-analyses. BMJ, 2011. 342: p. d549.

25. Higgins, J.P.T., et al., Cochrane handbook for systematic reviews of interventions. 2019: John Wiley \& Sons.

26. Borenstein, M., et al., Introduction to meta-analysis. 2011: John Wiley \& Sons.

27. Huguet, A., et al., Judging the quality of evidence in reviews of prognostic factor research: adapting the GRADE framework. Syst Rev, 2013. 2(1): p. 71. 